This item was submitted to Loughborough's Research Repository by the author.

Items in Figshare are protected by copyright, with all rights reserved, unless otherwise indicated.

\title{
Dry and wet nano-scale impact dynamics of rough surfaces with or without a
} self-assembled monolayer

PLEASE CITE THE PUBLISHED VERSION

PUBLISHER

Professional Engineering Publishing / @ IMECHE

VERSION

VoR (Version of Record)

LICENCE

CC BY-NC-ND 4.0

\section{REPOSITORY RECORD}

Teodorescu, M., and Homer Rahnejat. 2019. "Dry and Wet Nano-scale Impact Dynamics of Rough Surfaces with or Without a Self-assembled Monolayer". figshare. https://hdl.handle.net/2134/4701. 
This item was submitted to Loughborough's Institutional Repository (https://dspace.lboro.ac.uk/) by the author and is made available under the following Creative Commons Licence conditions.

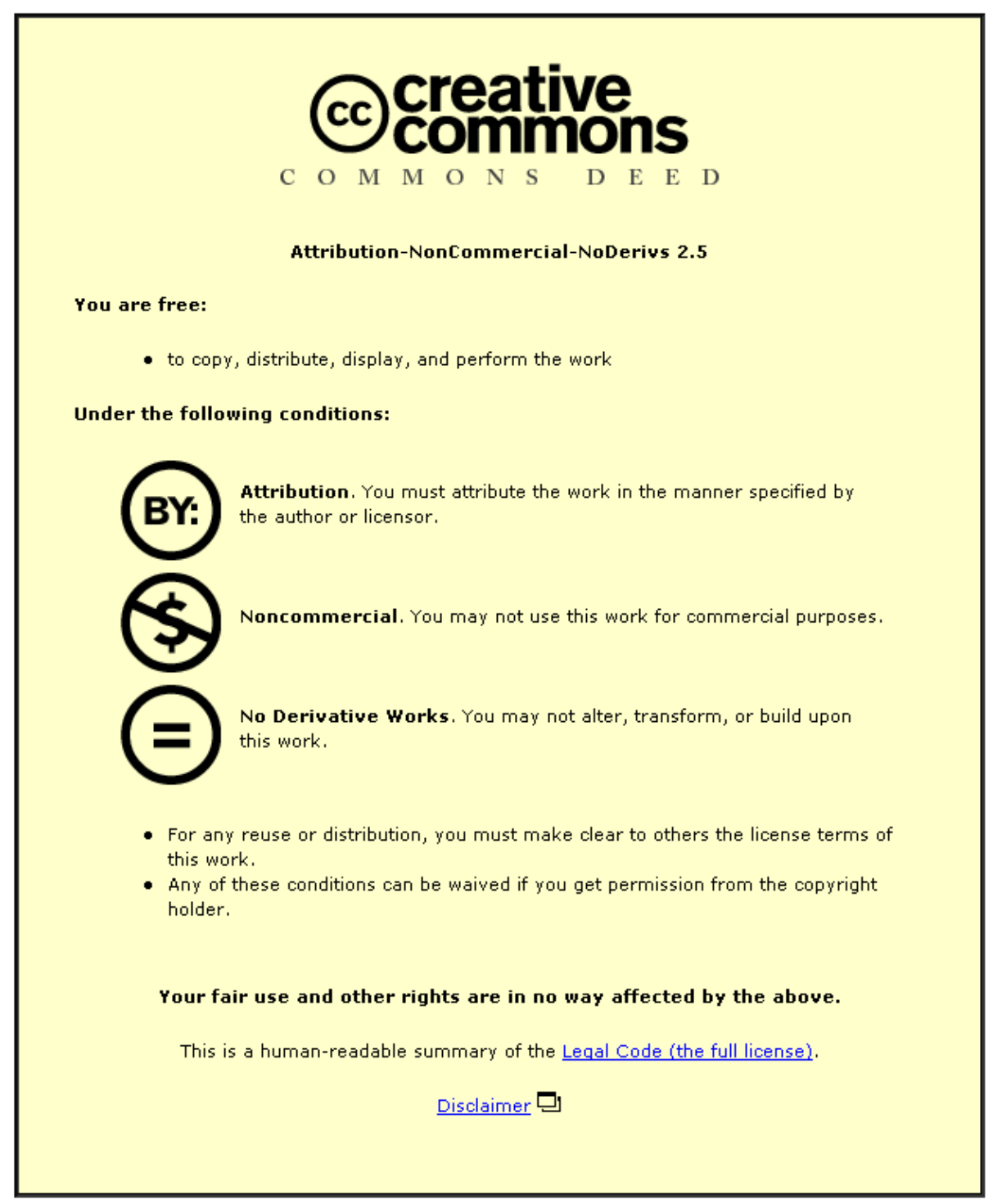

For the full text of this licence, please go to: http://creativecommons.org/licenses/by-nc-nd/2.5/ 


\title{
Dry and wet nano-scale impact dynamics of rough surfaces with or without a self-assembled monolayer
}

\author{
M Teodorescu ${ }^{1}$ and $\mathbf{H}$ Rahnejat ${ }^{2 *}$ \\ ${ }^{1}$ Department of Automotive Engineering, Cranfield University, Cranfield, UK \\ ${ }^{2}$ Wolfson School of Mechanical and Manufacturing Engineering, Loughborough University, Loughborough, UK
}

The manuscript was received on 7 March 2007 and was accepted after revision for publication on 19 October 2007.

DOI: $10.1243 / 17403499 J N N 101$

\begin{abstract}
In nanoscale conjunctions, molecular interactions cannot be ignored, nor surface energy effects, adhesion of surface asperities, or their stiction by any wetting action of an intervening fluidic media. Many experimental micro-electromechanical systems (MEMS) are prone to failure or malfunction due to structural degradation (wear and damage) of their load-bearing and power-transmitting conjunctions. Remedial solutions have been attempted by surface treatments, such as the introduction of self-assembled monolayers (SAMs) that adhere to the surfaces, and which are hydrophobic in nature. The physics of many of the contributing phenomena, such as adhesion, meniscus action, or electrostatics, are reasonably well understood, but their interactions under small-scale impacting condition is less investigated. The problem has a transient nature, owing to inertial dynamics, surface topography, attractive surface energy of asperities, and formation of any condensates. The interplay between these kinetics, using the established models, can be quite complex, particularly with regard to prediction of contact area and pull-off force in rebound of impacting pairs. The behaviour can vary from near classical Hertzian to that dominated by adhesion. The repercussions can be very significant in power transmission or load bearing for these small devices. This paper attempts to contribute to the growing understanding of contact behaviour in smallscale.
\end{abstract}

Keywords: nanoscale impact dynamics, adhesion, meniscus action, self-assembled monolayers, micro-electromechanical systems (MEMS)

\section{INTRODUCTION}

Wear and damage to contact conjunctions in loadbearing surfaces, such as meshing gear teeth of micro-electromechanical devices has become a major concern and a stumbling block in technological advances in the field $[\mathbf{1}, \mathbf{2}]$. The principal sources of the problem are adhesion of surfaces and ingression of moisture into the contact, both of which can cause damage to the rough-cut surfaces of the usual silica substrate. SAMs of long-chain molecules, such as octadecyltrichlorosilane (OTS) $[\mathbf{3}, 4]$, are made to adhere to the mating surfaces, which, due to their hydrophobic nature, are meant to inhibit formation of condensates. Furthermore, they tend to reduce

*Corresponding author: Wolfson School of Mechanical and Manufacturing Engineering, University of Loughborough, Loughborough, Leicestershire, LE11 3TU, UK. email: h.rahnejat@lboro. ac.uk the adhesion of mating rough surfaces. The kinetics in these very narrow conjunctions of molecular dimensions is quite complex and can comprise adhesion and meniscus action, as well as electrostatic repulsion, if a polar fluid forms a thin film. Adhesion of dry rough elastic contacting surfaces has been extensively studied and models have been developed, such as the Johnson-Kendoll-Roberts (JKR) model $[5,6]$ or the Derjaguin-Müller-Toporov (DMT) model [7]. Both these models are applicable for dry contact conditions and deviate from the classical Hertzian theory due to the fact that at small-scale separations attractive surface forces, promoted by adhesion, can become quite significant. This means that the Hertzian predictions for contact area become erroneous. The JKR and DMT models differ in the sense that the former is more applicable to less stiff asperities with strong adhesion, whilst the latter is best suited for stiffer asperities with weak adhesive contribution. There is of course a reasonably large intermediate 
range, which is not well described by either of the models, but has been tackled by the Maguis-Dugdale model [8].

In any study of small-scale rough elastic contacts, prediction of contact area, deflection, and pull-off force is thus very important, and dependent on asperity geometry, compliance, and surface attraction. In addition, if such surfaces are wetted by any film of a fluid, other kinetics should also be taken into account. Knowledge of contact mechanics behaviour of such surfaces and narrow conjunctions is continually evolving, as described by Carpick et al. [9], Berger et al. [10], Willemsen et al. [11], Tambe and Bhushan [12], Israelachvili [13], Al-Samieh and Rahnejat [14], Matsuoka and Kato [15], and Teodorescu et al. [16].

There has been less fundamental analysis for the case of impacting conjunctions in the nanoscale. AlSamieh and Rahnejat [17] presented results for complex kinetic interactions in such small gaps, but for lubricated cases, where the contact area is determined according to Hertz for smooth surfaces. When surface roughness is taken into account and dry conditions are promoted, such as in vacuum or in an inert atmosphere, the attractive adhesion force can become dominant according to asperity attributes (geometry and stiffness). However, under impacting conditions, deviations from Hertzian conditions due to attractive surface forces would be of a transient nature, and adhesion may not always be dominant, even for fairly compliant surfaces. This means that instantaneous conditions may not be best described by either JKR or DMT models, or by any specific intermediate model. In fact, Hertzian theory may be a good approximation for some aspects of rough elastic impacts. This paper investigates the rough elastic, very small-scale impact problem, starting from the JKR model. It further investigates the chance of menisci formation by condensation during such impacts. Accordingly, impact surfaces with and without SAMs are considered.

\section{DESCRIPTION OF THE PROBLEM}

Very small-scale devices include gear pairs (wheel and pinion), which are typically rough cut out of silicadeposited layers as power-transmitting pairs in microelectromechanical systems (MEMs). These gear pairs are very small: $20-100 \mu \mathrm{m}$ outside diameter. Therefore, contact of a typical meshing pair is of the order of sub-micrometre, with flank length of the order of $2 \mu \mathrm{m}$. In conventional gear pairs the separation (indicated by the undeformed gap $h_{r e f}$ in Fig. 1) would be two to three orders of magnitude smaller than the contact length. However, this is not the case in MEMS gears due to the width of the laser beam, cutting the gear profile, furnishing a separation of the order of a few micrometres, but at a relatively high

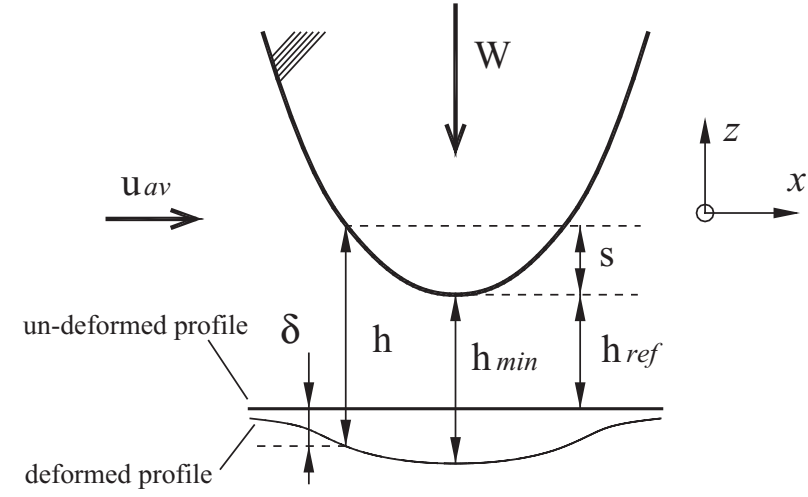

Fig. 1 Geometry of the gap

speed. Nevertheless, at such small-scale separations, surface energy effects play an important role to the extent that even under dry conditions asperity interaction is known to promote adhesion under elastostatic conditions [1, 7]. The extent to which adhesion plays a role depends on attributes of surface asperities, such as compliance and geometry. This means that Hertzian conditions are not adhered to where attractive interactions are considered. Under transient impact conditions, which are more relevant to the actual interactions, inertial dynamics can inhibit the role of adhesion, or, conversely, be affected by it. It is, therefore, necessary to commence from an asperity adhesion model and develop a contact model to predict the pull-off force to be used in small-scale impact dynamics. In this paper, the JKR model is chosen, which strictly means that the results would be appropriate to the case of relatively compliant asperities with strong adhesion contributions.

The use of this adhesion model precludes the other often observed problem that maintenance of an inert environment cannot be always assured, even if desired $[10,11]$. One problem is the ingression of moisture into the contact, with the result of menisci formation between the interacting asperity tips. The resulting meniscus force is also attractive and can lead to stiction. Under impacting condition, the effectiveness of a meniscus force depends on the impact time, which may be short lived, thus inhibiting the formation of menisci $[\mathbf{1 7}, \mathbf{1 8}]$. On the other hand, if the impact time is increased due to the action of attractive surface energy at small scale, the menisci may have adequate time to form. To guard against this eventuality, hydrophobic monolayers such as OTS are adhered to the surfaces.

Other forces can also contribute if the surfaces are molecularly smooth (very small asperity heights, of the order of tenths of a nanometre). The solvation force would be a main contributory factor due to the constraining effect of the surfaces as walls, even in the presence of air [13-16]. This mainly contributes a repulsive pressure. 


\section{HERTZIAN IMPACT FOR ELASTIC CYLINDERS}

Figure 1 shows the impact model, in which a rigid roller impacts a flat frictionless substrate of equivalent reduced elastic modulus, with an initial clearance of $h_{\text {ref. }}$. Due to impact, a localized deformation takes place, denoted by $\delta$, leading to a deformed gap shape, $h$, shown in the figure.

A relationship can be established between the impact force, contact footprint, and pressure distribution, based on the Hertzian theory as [19]

$$
W=\frac{\pi l E^{*}}{2 \ln \frac{2 l}{b}+1} \delta \approx K \delta ; \quad b=\sqrt{\frac{8 W R}{\pi E^{*} l}} ; \quad p=\frac{2 W}{\pi b l}
$$

where

$$
\begin{aligned}
& \delta=h_{\min }-h_{r e f} \\
& E^{*}=\left(\frac{1-v_{1}^{2}}{E_{1}}+\frac{1-v_{2}^{2}}{E_{2}}\right)^{-1}
\end{aligned}
$$

The maximum penetration depth and the impact time are obtained as [19]

$$
\begin{aligned}
& \delta_{\max }=\sqrt{M v^{2}\left\{2 \ln \frac{2 l}{b}+1\right\} / \pi l E^{*}} \\
& t_{\max }=\frac{2 \delta_{\max }}{v} \int_{0}^{1} \frac{1}{\sqrt{1-x^{2}}} d x=\frac{\pi \delta_{\max }}{v}
\end{aligned}
$$

The penetration in a Hertzian-type impact occurs in a symmetrical manner about the instant $t_{\max } / 2$. Thus, the instantaneous penetration can also be found, using forward differences as

$$
\delta_{i}=\delta_{i-1}+\left[\operatorname{sign}\left(t_{\max }-2 t\right)\right] \dot{\delta}_{i-1} \Delta t \quad 0 \leqslant t \leqslant t_{\max }
$$

Three important points should be noted. Firstly, the Hertzian impact theory assumes localized deformation (below modal response of the structure), thus the centre of impacting roller remains unaffected by body deformation. Secondly, the deformation is governed by the conversion of kinetic energy into elastic strain energy of the surface without any losses. Finally, there is no contribution due to friction or adhesion. These assumptions lead to the growth of deformation and contact footprint that do not correspond to attractive surface energy effects that are observed in smallscale approach of surfaces, such as those using atomic force microscope (AFM) measurements [9].

\section{ELASTIC IMPACT FOR ROUGH SURFACES}

The very proximate dry impact of rough surfaces is governed by a combination of inertial force and elastic deformation of asperities. Unlike the Hertzian impact,

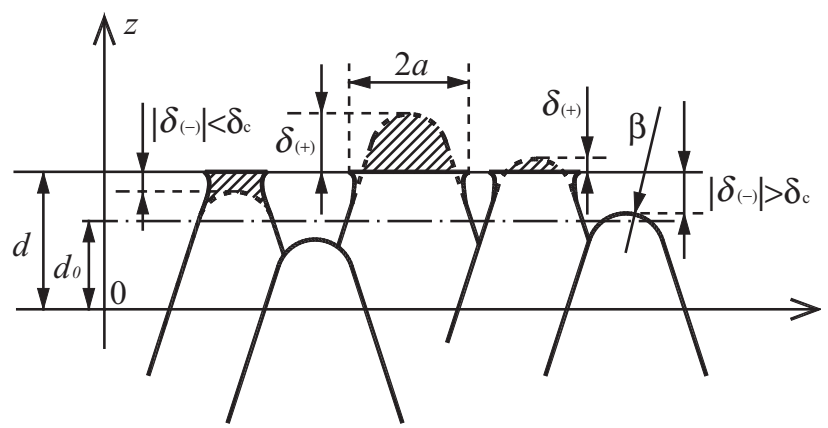

Fig. 2 Rough surface geometry

the rebound of the surfaces is affected by adhesion forces between adhered asperity tips. These are attractive forces, which resist separation. Thus, some work is expended to overcome adhesion, which affects the rebound height and the impact time, both of which deviate from the Hertzian predictions. Figure 2 is a representation of rough surface impact, in which some asperities show penetration, some are unaffected due to their smaller height, and others are stretched from their undeformed height during the rebound of an impacting solid. This stretching of asperities before pull-off is due to adhesion. $\delta_{c}$ is the maximum stretching of the asperity tips prior to pull-off (contact separation). Note that the real contact area comprises such asperity tip diameters $2 a$, being different to that predicted by the Hertzian theory.

\subsection{Adhesive contact of a single asperity}

An asperity contact model is proposed by Johnson and Sridhar [20], that comprises a deformation contribution according to Hertz and an adhesion component due to surface energy $\Delta \gamma$, thus the applied load is

$$
P=P_{0}-P_{a}=\frac{4 E^{*} a^{3}}{3 R}-\sqrt{8 \pi a^{3} \Delta \gamma E^{*}}
$$

where $\Delta \gamma=\gamma_{1}+\gamma_{2}+\gamma_{12}$, where for contact of same surface materials: $\gamma_{1}=\gamma_{2}$, and for fairly smooth surfaces (small asperities, such as with silica) one can assume that $\gamma_{12}=0$.

This net load and the corresponding elastic deflection can be non-dimensionalized as

$$
\left\{\begin{array}{l}
\overline{\bar{P}}=\overline{\bar{a}}^{3}-\sqrt{2 \overline{\bar{a}}^{3}} \\
\overline{\bar{\delta}}=\overline{\bar{\delta}}_{0}-\overline{\bar{\delta}}_{a}=3^{\frac{2}{3}} \overline{\bar{a}}\left(1-\frac{2 \sqrt{2}}{3} \overline{\bar{a}}^{-\frac{3}{2}}\right)
\end{array}\right.
$$

where

$$
\begin{aligned}
& \overline{\bar{a}}=a\left(\frac{4 E^{*}}{9 \pi \Delta \gamma \beta^{2}}\right)^{\frac{1}{3}} \\
& \overline{\bar{\delta}}=\delta\left(\frac{16 E^{* 2}}{9 \pi^{2} \Delta \gamma^{2} \beta}\right)^{\frac{1}{3}}
\end{aligned}
$$




$$
\overline{\bar{P}}=\frac{P}{3 \pi \Delta \gamma R}
$$

Fuller and Tabor [21] defined a pull-off force (to overcome adhesion) for a single semi-spherical asperity as

$$
P_{c}=\frac{3}{2} \pi \beta \Delta \gamma
$$

which corresponds to a maximum asperity stretching (extension) of

$$
\delta_{c}=\frac{1}{3 \beta}\left(\frac{9 \beta P_{c}}{4 E^{*}}\right)^{\frac{2}{3}}
$$

where

$$
\left\{\begin{array}{l}
\overline{\bar{P}}=\frac{P}{P_{c}}=\frac{P}{2 P_{c}} \\
\bar{\delta}=\frac{\bar{\delta}}{\delta_{c}}=\left(\frac{3}{4}\right)^{\frac{1}{3}} \frac{\delta}{\delta_{c}}
\end{array}\right.
$$

A point to note immediately is that clearly the loaddeflection characteristic is no longer the same as that for the contact continuum of classical Hertzian theory (see equation (1)).

Now substituting back into equation (4)

$$
\left\{\begin{array}{l}
\overline{\bar{P}}=2\left(\overline{\bar{a}}^{3}-\sqrt{2 \overline{\bar{a}}^{3}}\right) \\
\bar{\delta}=2.29 \cdot \bar{a}^{2}\left(1-\frac{2 \sqrt{2}}{3} \overline{\bar{a}}^{-\frac{3}{2}}\right) \rightarrow \\
\overline{\bar{P}}=f n(\bar{\delta})
\end{array}\right.
$$

which gives $\overline{\bar{P}}=f n(\overline{\bar{\delta}})$, the alternative to the Hertzian relationship in equation (1). This is a fairly complicated function, as shown in Fig. 3.

A convergence criterion should be used to determine numerically the function needed for the method.

\subsection{Adhesive contact of rough surfaces}

To extend the asperity contact model to the case of real contact of rough surfaces, it is necessary to assume the probability that an asperity has a height between $z$ and $z+d z$ above the plane defined by a

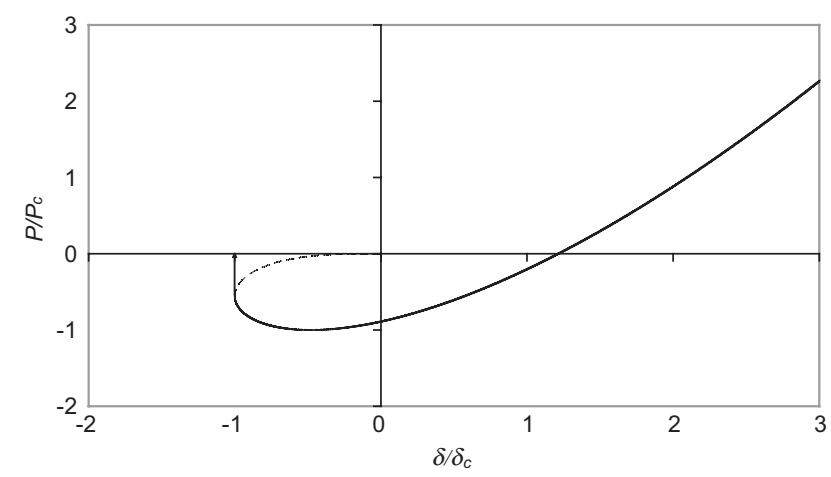

Fig. 3 Load deflection characteristics for the JKR model mean asperity height. A negative exponential distribution is usually assumed

$$
\phi(z)=\frac{1}{\sigma \sqrt{2 \pi}} \exp \left(-\frac{z^{2}}{2 \sigma^{2}}\right)
$$

where $\sigma$ is the root mean square of the composite rough surfaces. Thus, for a pair of rough surfaces approaching each other, the total contact force per unit area is the sum of all the compressive forces generated by the asperities whose height exceeds $d$ (see Fig. 2) [21]

$$
F=\frac{n P_{c}}{\sqrt{2 \pi}} \int_{0}^{\infty} f n\left(\frac{\Delta}{\Delta_{c}}\right) \exp \left\{-\frac{1}{2}(h+\Delta)^{2}\right\} d \Delta
$$

where

$$
\begin{aligned}
& \Delta=\frac{\delta}{\sigma} \\
& \Delta_{c}=\frac{\delta_{c}}{\sigma} \\
& h=\frac{d}{\sigma}
\end{aligned}
$$

Conversely, an attractive adhesive force may be imposed by asperities whose height is less than $d$ if they are stretched up to a limit of $\delta_{c}$, therefore

$$
F=\frac{n P_{c}}{\sqrt{2 \pi}} \int_{-L}^{\infty} f n\left(\frac{\Delta}{\Delta_{c}}\right) \exp \left\{-\frac{1}{2}(h+\Delta)^{2}\right\} d \Delta
$$

Here, the lower integration limit depends on the residual quantity $h-h_{\min }$ for any asperity, with $h_{\min }$ being the minimum separation (or maximum deformation, see Fig. 1). If $\Delta_{c}<h-h_{\text {min }}$, then the asperity is pulled-off, and the lower limit of the integral is $L=$ $\Delta_{c}$. On the other hand, there are other asperities still in contact and the limit has to be the residual $L=h-$ $h_{\min }>\Delta_{c}$.

For $n$ asperities, equations (10) and (11) give the rough surface contact force, which includes contributions from all the asperities in compression or through adhesion in separation. The integrals in equations (10) and (11) are then evaluated numerically.

\subsection{Meniscus and electrostatic forces for non contacting asperities}

When dry impact of a roller on a rough surface is considered (see section 3.4), ingression of moisture into the contact is insufficient to form a coherent film. Furthermore, it has been shown by Al-Samieh and Rahnejat [17] that for lubricated conjunctions of molecular dimensions contributions due to hydrodynamic action is negligible under impact conditions. 
They showed that the dominant force is the meniscus action. However, they did not consider the time necessary for menisci bridges to form during the usually very short impact time. For a semi-spherical asperity against an elastic semi-infinite solid, the meniscus force can be expressed as [22]

$$
F_{m}=-\frac{2 \pi R \gamma_{l v}\left(\cos \theta_{1}+\cos \theta_{2}\right)}{\lambda A_{m} \rho \ln \left(\frac{p_{s}}{p_{a}}\right)} \ln \left(\frac{w}{v_{a}}\right)
$$

Note that the negative sign indicates that the meniscus force is attractive, sticking the surfaces together. Considering the two surfaces to be dry before coming into contact, menisci can form by condensation from the surrounding atmosphere. Riedo et al. [22] computed the maximum height where a bridge can form as $h_{m w}=\ln \left(t / t_{a}\right)\left[\ln \left(p_{s} / p_{a}\right) A_{m} \rho\right]^{-1}$. They found the condensation time to be around $t_{a}=25 \mu \mathrm{s}$. Therefore, the meniscus force would act whenever the impact time becomes longer than the condensation time. During such a period, layers of water molecules adhere to the surface. With rough surfaces, having asperity heights greater than water molecules, a coherent film is not formed. The increasing depth of layers of water between the asperities gives rise to formation of menisci due to surface energy effects. With no coherent film of water any hydrodynamic effect due to squeeze-film action during impact of the roller may be discounted. A coherent film of sufficient thickness would be required for bulk properties of a fluid to characterize its behaviour (rather than surface energy effects), promoting hydrodynamic action.

\subsection{Impact dynamics}

A small elastic roller of radius $R$ is dropped onto a rough elastic substrate, having $n$ asperities of average height $\sigma$. The equation of motion is

$$
W-M g=M \ddot{z}
$$

where $W$ is the net impact force

$$
W=\int_{A} F d A+m F_{m}
$$

Note that the asperity adhesive force $F$ is per unit area of contact. There are $m$ asperities that form menisci. When the contact angle is obtuse, the meniscus force becomes positive (equation (12)). This corresponds to a hydrophobic surface, where a meniscus cannot be formed, thus $F_{m}=0$.

Now, from Fig. 1, it follows that the instantaneous height of the centre of the roller is

$$
z=R+h_{\text {ref }} \quad \text { or } \quad \ddot{z}=\ddot{h}_{\text {ref }}
$$

Thus, a solution is obtained for inertial dynamics in terms of $h_{r e f}$, using step-by-step integration.
The local gap at any position in the contact, given on a computational grid as $h_{i, j}$, is obtained as

$$
h_{i, j}=\frac{x_{i, j}^{2}}{2 R}+h_{r e f}+\delta_{i, j}
$$

where the local deformation is obtained as

$$
\delta_{i . j}=\frac{1}{\pi E^{*}} \sum \sum D_{i . j}^{k, l} p_{k . l}
$$

where $D_{i . j}^{k, l}$ is the influence coefficient matrix (see Hamrock [23]).

\section{RESULTS AND DISCUSSION}

To obtain nanoscale impact characteristics of MEMS devices with different surface topography, a series of simulations was carried out. A roller of radius $5 \mu \mathrm{m}$ and mass $0.6 \mu \mathrm{gm}$ made of silicon was released freely from a height of $100 \mathrm{~nm}$ to impact a nominally flat rough silicon surface. Different asperity heights were considered, effectively altering asperity compliance, thus the number of bounces and the corresponding rebound heights. As the asperity height was altered, the load-deflection characteristics changed. Consequently, the contribution due to adhesion would be different. Thus, the rebound behaviour altered (more or less of the impact kinetic energy is lost due to adhesion). The progressively decaying and reducing number of rebounds provided an indication of deviation from idealized Hertzian impact condition, where the principle of conservation of energy is upheld.

In practice, the loss of mechanical energy and lower rebound height would be of interest in order, for example, not to initiate load reversals in gearings. The loss of energy would be mainly in the form of heat, which would not usually be of concern due to good thermal stability of silicon. However, as the effect of adhesion becomes more significant and impact time prolongs, it is expected that a combination of adhesion and meniscus force would inhibit rebound of the surfaces. More energy would be required to overcome stiction, which in practice will be provided by the driving forces in the mechanism. This can lead to inefficiency or plastic deformation of asperities or both. Therefore, hydrophobic SAMs such as OMT are adhered to the substrate material (usually silicon, alumina, or mica) in order to demote the chance of menisci formation, as well as act rather like compliant asperities with insignificant adhesion.

A comparison of Figs 4(a) and 4(b) shows the effectiveness of SAM in reduction of adhesion. Figure 4(a) shows the contact behaviour of a rough silicon surface in compression (deformation) $\left(p_{L=0}\right)$, followed by separation $\left(p_{L=-1}\right)$. The two characteristics are not coincident, owing to adhesive behaviour during the 


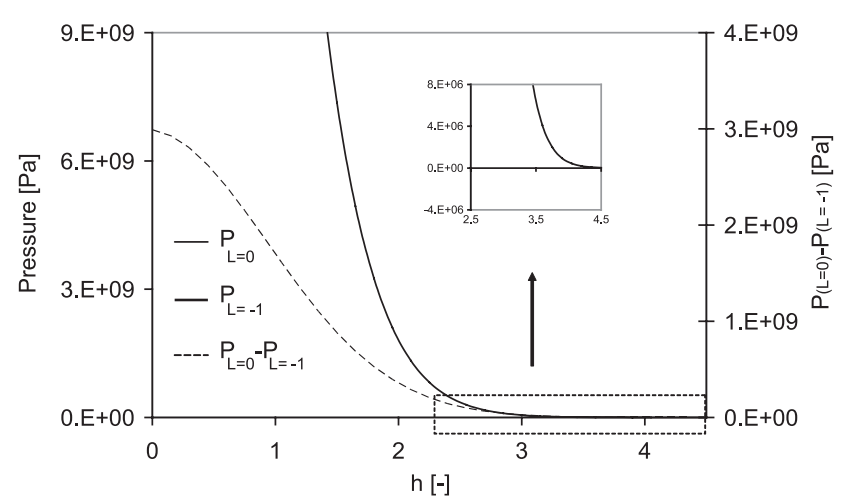

(a) Silica surfaces

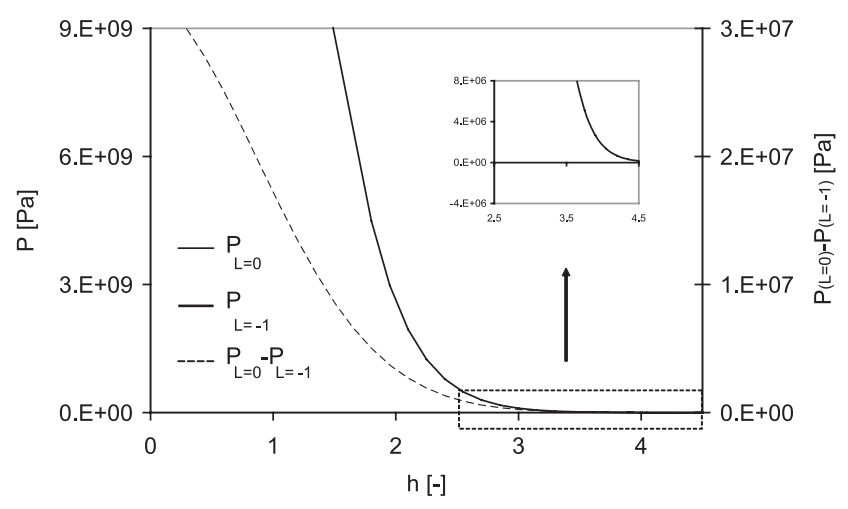

(b) SAM on silica surfaces

Fig. 4 Contact characteristics of rough adhesive and hydrophobic monolayer surfaces

pull-off (as described previously). Negative pressures indicate dominant adhesive behaviour. The important point to note is that the deviation from conformance of the characteristics in deformation and extension (shown by $p_{L=0}-p_{L=-1}$ ) represents a significant pressure, which would account for principal loss of rebound energy. On the contrary, Fig. 4(b), showing the same characteristics for SAM (based on OTS), indicates significantly lower differences.

Figure 5(a) compares the rebound characteristics of smooth, rough, and OTS- covered silicon surfaces as the aforementioned roller (with or without an OTS monolayer) is released from a height of $100 \mathrm{~nm}$ to impact them. Clearly, when the Hertzian theory is used the assumption of a smooth frictionless surface is implicit. The Hertzian theory is based on conservation of mechanical energy (kinetic and potential). Thus, the rebound height is the same as the release height, and there is no limit to the number of bounces. When the rough nature of the surface is taken into account (in this case with an average height of $6 \mathrm{~nm}$ ), and under dry impact condition, the loss of energy in rebound is in line with the characteristics in Fig. 4(a). Thus, the rebound height progressively reduces with successive impacts, as shown in Fig. 5(a). The numbered sharp vertical lines indicate successive impact durations. These are magnified in Fig. 5(b).

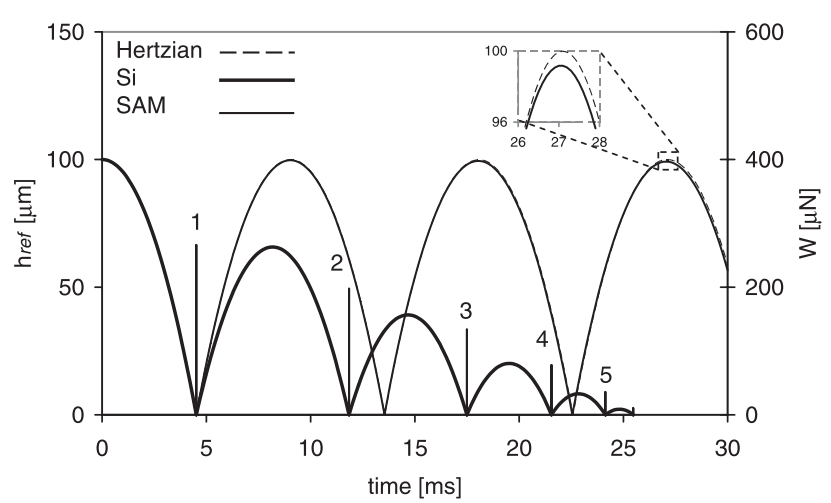

(a) Silica - Silica and SAM covered Silica-SAM covered Silica impacts

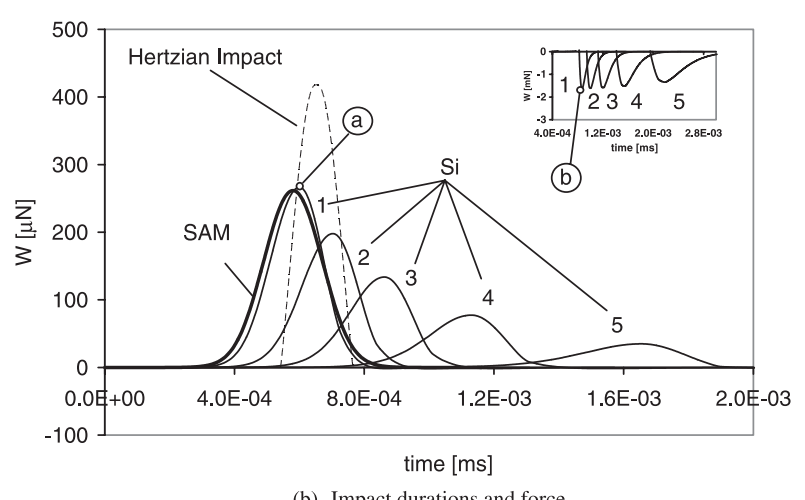

Fig. 5 Small-scale impact characteristics

In Fig. 5(a), the impact behaviour of the OTS monolayer on the silicon substrate closely follows the Hertzian model (see the inset in Fig. 5(a)), with a very gradual loss of rebound potential energy. This is rather fortunate, making contact mechanics predictions for such surfaces, increasingly used in practice, a rather simple task. However, it should be noted that the current analysis does not take into account any friction, which occurs in oblique impacts, nor plastic deformation or wear of the monolayer.

Referring to Fig. 5(b), the impact duration in the Hertzian-type impact is shorter with a higher impact force than the other cases (first impact only shown). This is expected due to no loss of kinetic energy (coefficient of restitution being unity). With rough impact of silica, the impact time is increased, because some of the asperities experience adhesion and cause delayed rebound. Some of the kinetic impact energy is lost in this process, resulting in a lower strain energy to be converted back into rebound kinetic energy (coefficient of restitution $<$ unity). This can be noted mathematically using Euler's formula $E=\int W d \delta$, where the net force $W$ given by equation (14) is, therefore, much less than that for a Hertzian impact given by equation (1). The depth of deformation is consequently higher for a Hertzian impact. Figure 5(b) shows the progressively reducing net impact force, thus the rebound energy. Consequently, the impact 

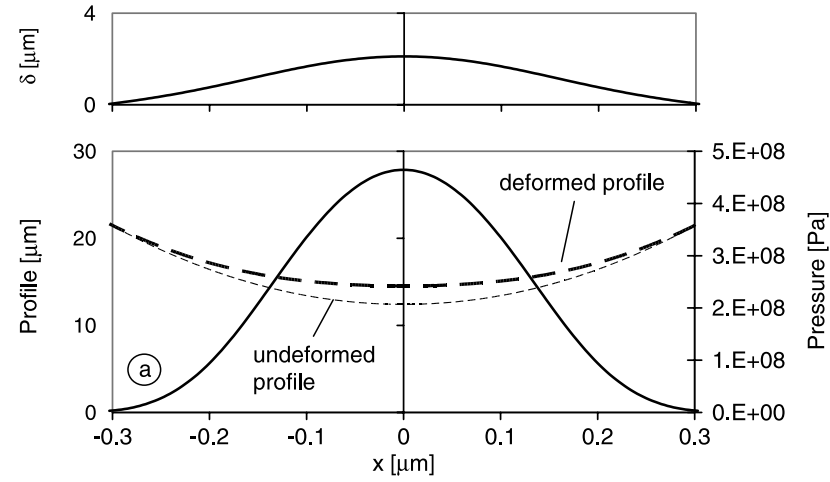

(a) At maximum penetration
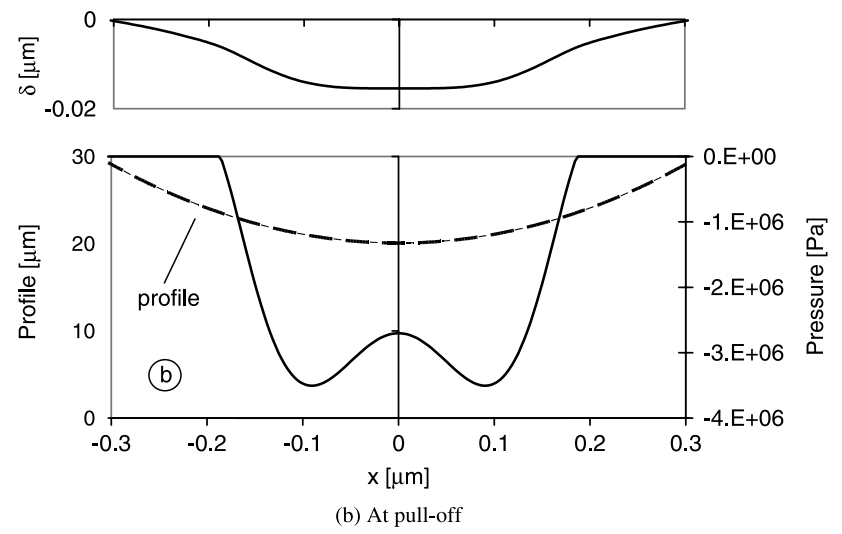

Fig. 6 Deformation characteristics of rough elastic solids through small-scale impact and rebound

time lengthens with progressive impacts with decreasing $W$. The inset in Fig. 5(b) shows an increasing contribution from adhesion with subsequent bounces. Eventually, an insufficient energy exists for the roller to leave the surface of the silicon substrate. Using the analogy of projectiles, there is a limiting escape velocity from any surface for an impacting elastic rough solid in small-scale. This would also depend on wetness of the surface. Note that for the results presented in Fig. 5, there is no effect from meniscus, because the impact durations are below water condensation time (see section 3.3). This situation alters with the lower initial impact kinetic energy, surface topography and material properties. With SAM, the behaviour is quite similar to the real rough silicon impact, but contribution due to adhesion is insignificant, thus sufficient energy exists for the impacting solid to lose rebound height only very gradually over many bounces.

Figure 6 shows the deformation behaviour of rough elastic silicon at two distinct instances through impact and rebound, pertaining to the instance of maximum deflection (marked by $a$ in Fig. 5(b)) and at the instance of rebound/pull-off (marked by $b$ in the inset to the same figure). The conditions at maximum deflection are shown in Fig. 6(a). The surface deformation $\delta$ corresponds to the difference between the global undeformed profile, and that

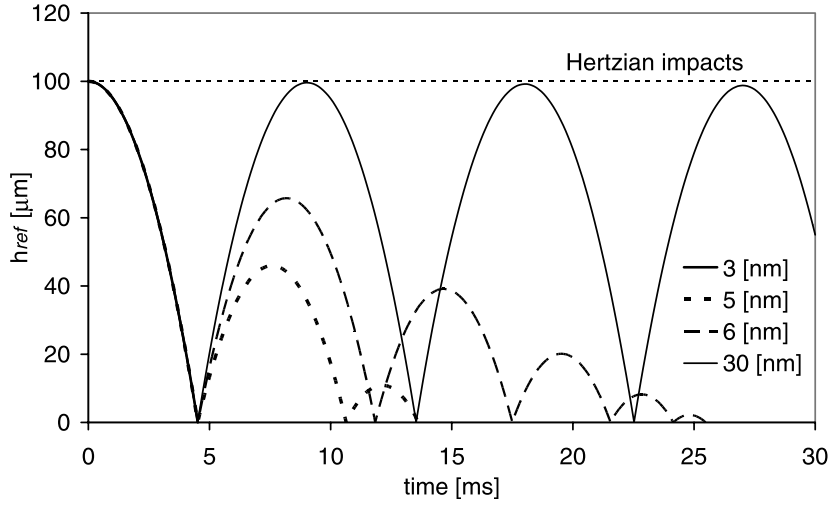

Fig. 7 The influence of asperity height in small-scale impact dynamics of silica

shown by the deformed shape of the roller. The corresponding pressure distribution is also shown. At pull-off, there is a small extension of the surface as shown in Fig. 6(b), and the corresponding pressure profile indicates a suction effect caused by adhesion. The work of adhesion corresponds to the energy expended to overcome this suction effect. Note that the area under the pressure distribution is the net contact load and the energy expended in pull-off is given by the Euler relationship $E_{\text {pull-off }}=\int F d \Delta$, where $F=\int p d x$. Clearly, if the meniscus action also operates, the work of adhesion is increased due to $F_{m}$. For a given material as the surface topography is varied, the work of adhesion alters. To observe the influence of topography, the average roughness height is altered in the range $3-30 \mathrm{~nm}$ and the same impact conditions are used as before. The Hertzian rebound height is indicated by the dashed line in Fig. 7, which is independent of surface roughness and distribution. For the ubiquitous asperities, the average height is varied, affecting the rebound characteristics as shown in the figure. Shorter asperities of the same material act stiffer in extension (produce a greater adhesion force), thus both the number of bounces and the rebound heights are reduced. In fact, for the case of silicon, the asperity height of $3 \mathrm{~nm}$ Ra shows no rebound. For the case of $30 \mathrm{~nm}$ $\mathrm{Ra}$, the rebound height almost conforms to the Hertzian condition, with a very slow decay rate. Thus, at the first glance one might conclude that rough surfaces with increasing asperity heights tend to Hertzian condition under frictionless impact. This contradicts the tenets of Hertzian impact theory (valid for smooth surfaces), where one would expect the contrary. Therefore, one should further investigate this point.

Referring to equation (2), note that the relationship between the impact time and rebound velocity is hyperbolic for Hertzian conditions, where $v t_{\max }=$ $\pi \delta_{\max }=C$, where $C$ is a constant for a given impact condition. On the logarithmic scale (as shown in 


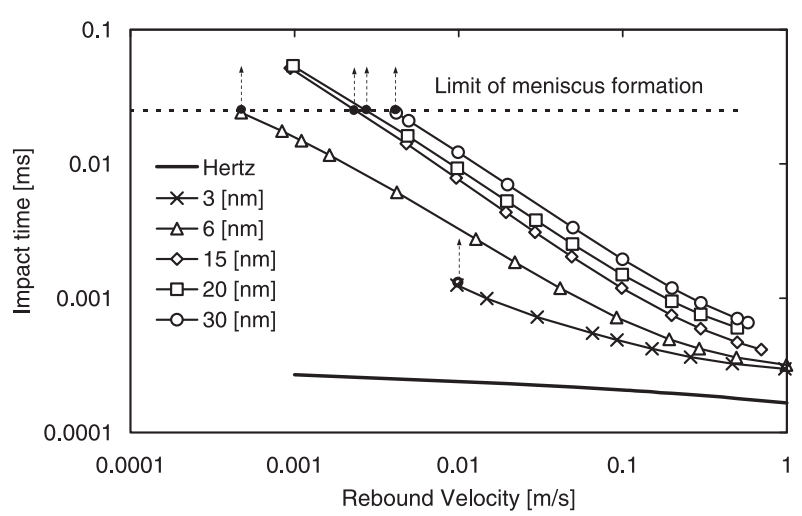

(a)

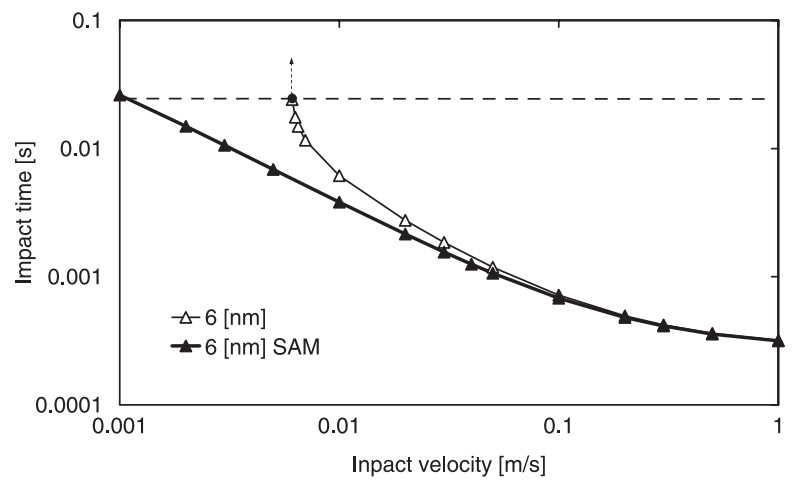

(b)

Fig. 8 Impulsive characteristics of rough elastic silica surface

Fig. 8(a)), this would yield a linear relationship of a decreasing slope with increasing rebound velocity. The limits of this relationship are:

(i) at rest (yielding a Hertzian elastostatic contact), and

(ii) at a limiting impact velocity, abrogating the Hertzian small-strain assumption (resulting in inelastic deformation when the sub-surface maximum shear stress exceeds the maximum Hertzian pressure, Tresca criterion $\tau_{\max }>0.31 p_{\max }$ ).

Thus, for Hertzian impacts principle of conservation of momentum is conserved in the totally elastic impact. Any deviation from linearity in Fig. 8(a) constitutes loss of rebound momentum due to adhesion, whilst the conditions still remain elastic. A surface with a shorter average asperity height exhibits stiction at a higher rebound velocity (i.e. demands a higher escape velocity). These limiting velocities are shown by vertical arrows in the figure, below which no rebound occurs. As the asperity height is increased, there is a greater chance of rebound with a shorter impact time. This shows that the impact behaviour of smoother surfaces, in fact, tend to Hertzian conditions, as would be expected. However, it never conforms to it until the effect of adhesion is fully removed (i.e. no asperity interactions/adhesion). In nanoscale, such conditions correspond to molecularly smooth surfaces in mutual approach, where the presence of any moisture can lead to a host of other phenomena, such as electrostatic repulsion, solvation, van der Waal's interactions, and even formation of a hydrodynamic film, depending on velocity of approach and applied load [15-17]. With higher average asperity heights, the rebound velocity decreases with increasing impact time, until the latter becomes long enough for menicus action around the interacting asperity tips, and when both $\theta_{1}$ and $\theta_{2}$ remain acute. This limiting impact time $(25 \mu \mathrm{s})$ is shown by the horizontal dotted line in the figure. Thereupon, increased resistance due to adhesion and meniscus action prohibits any further rebounds.

The rebound characteristics of a hydrophobic monolayer (such as OTS) adhered to the silicon surface can be compared to that of the exposed silicon surface itself, as shown in Fig. 8(b). It can be seen that with a monolayer of $2 \mathrm{~nm}$ thickness (used in this example) on the silicon surface of $6 \mathrm{~nm}$ Ra roughness, the characteristics deviate as the impact velocity is reduced. Thus, the exposed silicon surface shows progressively longer impact times with reduced impact momentum compared to that with a layer of OTS. This is due to the increasing role of adhesion at a reduced impact height. Ultimately, the exposed surface would stick at the limit of adhesion governed here by meniscus action in a larger gap than that with a monolayer. This has an important practical implication since, for example, MEMS gears (like all gears) have backlash and a larger limiting backlash is required for the unprotected rough surface (without a monolayer) for safe operation in presence of adhesion and meniscus action [1]. A larger minimum limiting gap corresponds to a higher impact energy, thus a greater chance of fatigue spalling by inelastic deformation.

\section{CONCLUSION}

It has been shown that the impact behaviour of nanoconjunctions deviates from the classical Hertzian impact theory due to adhesion of surface asperities. The adhesive characteristics depend on the model used. In the case of the JKR model, a strong adhesion contribution is predicted for fairly compliant asperities, resulting in longer impact times and lower rebound velocities with higher average asperity heights. Thus, impact characteristics of smoother surfaces tend towards Hertzian behaviour, but never conform to it due to adhesion at close range. When hydrophobic monolayers are used to cover such rough surfaces, the effect of adhesion is largely removed and impact conditions tend to Hertzian. This has the added advantage of inhibiting the formation of condensates, which can form between asperity tips at close range and further promote stiction. However, it is necessary to extend the current 
mathematical model to include the effect of friction (particularly for oblique impacts) and plastic deformation of asperities and monolayers through ploughing action.

When the surfaces are exposed to environment, formation of menisci around asperity tips become important, and depend on the contact angle for a particular surface and the impact time versus time for water condensation. Thus, with increased impact time (lower kinetic energy and increased adhesion), the meniscus action can act and cause stiction of surfaces. This problem can occur quicker with reduced asperity heights. However, the presence of moisture and relative motion of surfaces can also bring about formation of very thin polar films that can generate electrostatic and hydrodynamic pressures. At very close range, with molecularly smooth surfaces reordering of water molecules near the surfaces can also cause solvation pressure [14-16]. The current model should be extended to include such effects, which may be prevalent in practice.

\section{REFERENCES}

1 Tanner, D. M., Smith, N. F., Irwin, L. W., Eaton, W. P., Helgesen, K. S., Clement, J. J., Miller, W. M., Walraven, J. A., Peterson, K. A., Tangyunyong, P., Dugger, M. T., and Miller, S. L. MEMS Reliability: Infrastructure, Test Structures, Experiments, and Failure Modes, 2000 Sandia Report, SAND2000-0091, Laboratory Directed Research and Development (LDRD) program, Sandia National Laboratories, USA.

2 Hsu, T. R. MEMS and Microsystems: Design and Manufacture. 2002 (McGraw-Hill, Boston).

3 Maboudian, R., Ashurst, W. R., and Carraro, C. Selfassembled monolayers as anti-stiction coatings for MEMS: characteristics and recent developments. Sensor Actuators, 2000, 82, 219-223.

4 Reedy, E. D., Jr., Starr, M. J., Jones, R. E., Flater, E. E., and Carpick, R. W. Contact modeling of SAM-coated polysilicon asperities, In Proceedings of the 28th Annual Meeting of the Adhesion Society. Mobile, AL 13-16 Feb. 2005.

5 Johnson, K. L., Kendall, K., and Roberts, A. D. Surface energy and the contact of elastic solids. Proc. Roy. Soc., Series A, 1971, 324(1558), 301-313.

6 Johnson, K. L. Adhesion and friction between a smooth elastic spherical asperity and a plane surface. Proc. $R$. Soc. Lond. A, 1997, 453, 163-179.

7 Derjaguin, K. L., Muller, V. M., and Toporov, Y. P. Effect of contact deformation on the adhesion of particles. J. Colloid Interface Sci., 1971, 53(314), 314-326.

8 Maugis, D. Adhesion of spheres: the JKR-DMT transition using a dugdale model. J. Colloid Interface Sci., 1992, 150, 243-269.

9 Carpick, R. W., Ogletree, D. F., and Salmeron, M. A general equation for fitting contact area and friction vs load measurements. J. Colloid Interface Sci., 211, 1999, 395-400.
10 Berger, C. E. H., van der Werf, K. O., Kooyman, R. P. H., de Grooth, B. G., and Greve, J. Functional group imaging by adhesion AFM applied to lipid monolayers. Langmuir, 1995, 11, 4188-4192.

11 Willemsen, O. H., Snel, M. M. E., Cambi, A., Greve, J., de Grooth, B. G., and Figdor, C. G. Biomolecular Interactions Measured by Atomic Force Microscopy. Biophys., 2000, 79, 3267-3281.

12 Tambe, N. S. and Bhushan, B. Friction model for the velocity dependence of nanoscale friction. Nanotech., 2005, 16, 2309-2324.

13 Israelachvili, J. N. Intermolecular and Surface Forces, 1992, (New York: Academic).

14 Al-Samieh, M. F. and Rahnejat, H. Ultra-thin lubricating films under transient conditions. J. Phys. D: Appl. Phys., 2001, 34, 2610-2621.

15 Matsuoka, H. and Kato, T. An ultra-thin liquid film lubrication theory - calculation method of solvation pressure and its applications to EHL problem. Trans. ASME, J. Tribology, 1997, 119, 217-226.

16 Teodorescu, M., Balakrishnan, S., and Rahnejat, $\mathbf{H}$. Physics of ultra-thin surface films on molecularly smooth surfaces. Proc. IMechE, Part N: J. Nanoengineering and Nanosystems, 2006, 220(1), 7-19.

17 Al-Samieh, M. F. and Rahnejat, H. Physics of lubricated impact of a sphere on a plate in a narrow continuum to gaps of molecular dimensions. J. Phys. D: Appl. Phys., 2002, 35, 2311-2326.

18 Bhushan, B. Handbook of Micro/Nanotechnology, 2nd edition. 1999 (CRC Press, Boca Raton, Florida).

19 Teodorescu, M., Votsios, V., and Rahnejat, H. Multiphysics analysis for the determination of valve train characteristics. Proc. IMechE, J. Auto. Engng., 2005, 219(9), 1109-1117.

20 Johnson, K. L. and Sridhar, I. Adhesion between a spherical indenter and an elastic solid with a compliant elastic coating. J. Phys. D: Appl. Phys., 2001, 34, 683-689.

21 Fuller, K. N. G. and Tabor, D. The effect of surface roughness on the adhesion of elastic solids. Proc. Roy. Soc., Series A, 1975, 345(1642), 327-342.

22 Riedo, E., Lévy, F., and Brune, H. Kinetics of capillary condensation in nanoscopic sliding friction. Phys. Rev. Lett., 2002, 88(18).

23 Hamrock, B. J. Fundamentals of fluid film lubrication. 1994, (McGraw-Hill International Editions, New York, London, New Mexico).

\section{APPENDIX}

\section{Nomenclature}

a contact area of an asperity

$A \quad$ apparent contact area

$A_{m} \quad$ meniscus area

$b \quad$ contact semi-half-width

$D_{i . j}^{k, l} \quad$ the influence coefficient matrix

$E_{\text {pull-off }}$ strain energy

$E_{1,2} \quad$ Young's elastic modulus of surfaces

$E^{*} \quad$ reduces elastic modulus 


\begin{tabular}{|c|c|c|c|}
\hline$F$ & Force on an asperity & $t_{a}$ & condensation time \\
\hline$F_{\mathrm{m}}$ & meniscus force per asperity contact & $t_{\max }$ & contact time \\
\hline$g$ & gravitational acceleration & $v$ & impact velocity \\
\hline$h$ & local gap & $v_{a}$ & critical velocity of water condensation \\
\hline$h_{\min }$ & minimum elastic thickness & $w$ & local approach velocity \\
\hline$h_{m w}$ & molecular thickness of water & $W$ & impact force \\
\hline$h_{\text {ref }}$ & minimum rigid gap & $x$ & transverse contact direction \\
\hline$l$ & roller length & $z$ & mean asperity height \\
\hline$m$ & number of menisci bridges & & \\
\hline$M$ & roller mass & $\beta$ & asperity tip radius \\
\hline$n$ & number of asperities per unit area & $\gamma_{1,2}$ & surface energies \\
\hline$p$ & contact pressure & $\gamma_{12}$ & interfacial surface energy \\
\hline$p_{a}$ & atmospheric pressure & $\gamma_{\mathrm{lv}}$ & liquid-vapour interfacial surface tension \\
\hline$p_{\mathrm{k}, l}$ & $\begin{array}{l}\text { pressure at any location } k, l \text { in the computa- } \\
\text { tional grid. }\end{array}$ & $\begin{array}{l}\delta \\
\delta_{c}\end{array}$ & $\begin{array}{l}\text { local deformation } \\
\text { maximum asperity stretching }\end{array}$ \\
\hline$p_{\max }$ & maximum Hertzian pressure & $\delta_{i, j}$ & elastic deformation at any position $i, j$ in the \\
\hline$p_{s}$ & saturation pressure & & computational grid \\
\hline$P$ & applied load on an asperity & $\theta_{1,2}$ & contact angles \\
\hline$P_{O}$ & Hertzian contact force per asperity & $\lambda$ & typical lateral gap of interacting asperities \\
\hline$P_{a}$ & force of adhesion on an asperity & $\rho$ & density of water \\
\hline$P_{c}$ & pull-off force & $\sigma$ & RMS surface roughness \\
\hline$R$ & roller radius & $\tau_{\max }$ & maximum shear stress \\
\hline$t$ & time & $v_{1,2}$ & Poisson's ratio \\
\hline
\end{tabular}

